\title{
Avoiding Simple Mistakes Commonly Seen in JSGS Manuscripts (Part 2)
}

\author{
Takako Kojima $^{1)}$ and J. Patrick Barron ${ }^{2)}$ \\ 1) Department of International Medical Communications, Tokyo Medical University \\ 2) Tokyo Medical University
}

This article is a continuation of the previous article wherein we, as the English editors of the journal, address some of the mistakes we commonly find in the Japanese Journal of Gastroenterological Surgery (JSGS Journal) abstracts.

\section{1) "case" vs. "patient"}

We often see sentences such as:

The case underwent surgery.

This is incorrect because a case is a particular instance of a disease, whereas a patient is a particular person under medical care. Furthermore, a case is something that we can evaluate, document, or report. A patient, on the other hand is examined, undergoes testing, and is treated ${ }^{1}$. With this in mind, it would be wrong to use case and underwent together in a sentence. Therefore the following usage is recommended:

The patient underwent surgery.

The case was evaluated for its results.

Exceptions would be for case-control studies, in which situation it would be appropriate to say patients in the case group.

\section{2) Placing classifications}

It is better to place the classifications, such as 'grade 1' or 'stage 1' before the tumor.

For example:

Correct duodenal grade 1 neuroendocrine tumor

Incorrect duodenal neuroendocrine tumor Grade 1

If the classification is placed at the end, it gives no specific meaning to the duodenal neuroendocrine tumor, and could (theoretically at least) be interpreted to mean something unrelated, such as Grade 1 in elementary school. Since it is the tumor that has been classified, it should be placed before the tumor so that it acts as an adjective.

It would, however, be acceptable to place 'grade 1' at the end if used in parentheses.

For example:

Duodenal neuroendocrine tumor (grade 1).

Whether the "g" of the word "grade" should be capitalized or not depends on how it appears in the original classification. 


\section{3) "compared to" vs. "compared with"}

This is quite difficult for a non-native speaker of English (and even native speakers of English). The general idea is that something or someone is compared with another when the goal is to find what is similar or different from the other, both belonging to the same group, e.g. patients.

For example:

We compared X-ray findings of patient A with patient B.

Here, we are trying to examine the similarities and differences between drugs A and B.

On the other hand, when something is compared to another, it is being used as a metaphor.

For example:

He compared the sun to the chariot of the gods.

This suggests that it, the sun, can be taken as, or interpreted as, the chariot of the gods.

The American Medical Association Manual of Style (AMA) gives another relevant example:

"Few medical discoveries can compare to the discovery of penicillin."2)

This sentence is stating that there are only a few significant medical discoveries which can be compared to or are like the monumental achievement of the discovery of penicillin.

\section{4) Use of eponyms}

The definition of eponym is "one for whom or which something is or is believed to be named"3). There are a number of drugs and diseases that are derived from eponyms such as Parkinson disease and Down syndrome.

Although it was common to see these listed as Parkinson's disease or Down's syndrome, authors should be aware of the fact there is an ongoing debate on the use of such possessive forms of eponyms, and the use of the nonpossessive (without using apostrophe - 's) form of eponyms is becoming more and more the standard ${ }^{4}$. The AMA states that societies such as the National Down Syndrome Society in the U.S. prefer to use Down syndrome rather than Down's syndrome for the reason that the syndrome does not actually belong to anyone. Furthermore, major dictionaries such as Stedman's Medical Dictionary, as well as Dorland's Illustrated Medical Dictionary, recommend the use of nonpossessive forms of eponyms, demonstrating the preference or standard trend in the usage of nonpossessive eponymous terms.

\section{5) Misuse of 'diameter'}

We often come across expressions such as:

The tumor was $7 \times 3$ in diameter.

This is incorrect as the word 'diameter' refers to the maximum distance from one side of a perfect circle to the other. Therefore it would not be possible to have a diameter of a single tumor which is not a perfect circle, and it would be impossible to have two different diameters of one entity. The correct usage in the example of a circle would be:

The tumor was $5 \mathrm{~cm}$ in diameter.

or if specific measurements are to be given,

The tumor was $7 \times 3 \mathrm{~cm}$. 
Perhaps because of the similarity in spelling, diameter is frequently mistakenly used instead of "dimension".

\section{Ending Message}

We hope that the common mistakes covered in both Part 1 and Part 2 of our article Avoiding Simple Mistake Commonly Seen in JSGS Manuscripts, will help our future authors improving their skills in medical writing. Future series on other topics are planned.

\section{References}

1) American Medical Association. AMA manual of style: a guide for authors and editors. 10th ed. New York: Oxford University Press; 2007. Chapter 11, Correct and Preferred Usage of Common Words and Phrases; p. 388.

2) American Medical Association. AMA manual of style: a guide for authors and editors. 10th ed. New York: Oxford University Press; 2007. Chapter 11, Correct and Preferred Usage of Common Words and Phrases; p. 390.

3) Merriam-Webster Dictionary and Thesaurus [Internet]. Available from: http://www.merriam-webster.com/dictionary/eponym. Accessed September 2, 2015.

4) American Medical Association. AMA manual of style: a guide for authors and editors. 10th ed. New York: Oxford University Press; 2007. Chapter 16, Eponyms; p.777-8. 